\title{
Quadruple or triple action creams for insect venom treatments
}

EDITORIAL

Pardis Tabaee Damavandi | 07/06/2021

Insect stings ${ }^{1}$ are very common in the summer season and the extent of the damage caused by them can range from mild rashes, with swollen and raised skin bumps to downright debilitating chronic diseases, such as Lyme Disease ${ }^{2}$, which for years has not had a cure, or worse it may result in neurotoxic poisoning (thinking of some species of spiders and scorpions ${ }^{3}$ ) which can turn fatal quickly; even just a wasp sting can induce anaphylactic shocks to extents that can be life-threatening if not managed timely.

\section{THE CHALLENGE}

Presently, topical creams, gels, paste or ointments are the preferred route of administration for the treatment of the rashes, however, although some creams are available with multiple active ingredients, they are not designed specifically for insect sting relief and the necessity to include more than one active ingredient for this particular purpose can help managing insect bites or stings timely and effectively, but also prophylactically. Clearly, this alone may not suffice and should be accompanied by routine observations of the patients, who may develop "adverse reactions" to stings beyond the twenty-four hour period from the sting or bite.

\section{A POSSIBLE SOLUTION}

Generally, depending on the insect puncture, which is means of introduction of foreign bodies, including stingers, toxins and microbes, it can be expected that a combination of multiple active principle ingredients can be included in a topical emulsion.

Firstly, an antihistaminic, i.e. Cetirizine, could help in the case of particular stings or bites that could cause an elevated response to the point of anaphylactic shock, as in the case of wasp stings, where the body may struggle to "battle" the wasp's venom. 
Secondly, the employment of lidocaine which anesthetizes the area and removes pruritus and pain could also be beneficial, some creams already have benzocaine associated with one more active ingredient (e.g. zinc oxide and hydrocortisone). Thirdly, a strong antiseptic or antibiotic, which can range from a "milder" macrolide to a stronger tetracycline, such as doxycycline, which could be particularly helpful in infectious tick bites, and perhaps mildly prophylactic, could also be supplementary.

Ultimately, the usage of a homeopathic (herbal) remedy within the emulsion, which is perfect in the treatment of wounds, and is also lenitive in its action could be of aid, particularly the presence of herb Arnica Montana L., although this is contraindicated in patients who suffer from ragweed allergies; an alternative could be that of Matricaria Chamomilla L., otherwise known as the common chamomile, or Melissa Officinalis L., renown as lemon balm. Peppermint (Mentha Piperita L.) itself which has refreshing, soothing-lenitive properties could also be added in the preparation.

Although optimal formulation is always the target in the case of emulsion-liquid topicallocal preparations, they can be produced industrially effortlessly and they can also stimulate medicinal plant cultivation.

\section{CONCLUSION}

A quadruple action topical formulation could be beneficial in the "supervision" of insect stings, or bites, and mildly prophylactic upon patient presentation, prior to more drastic drug management.

\section{Author declaration}

No conflicts of interest to disclose.

\section{REFERENCES}

1. 1. Ellis AK, Day JH. Clinical reactivity to insect stings. Curr Opin Allergy Clin Immunol. 2005;5(4):349-354.

2. Piesman, J., Hojgaard, A., Ullmann, A. J., \& Dolan, M. C. (2014). Efficacy of an experimental azithromycin cream for prophylaxis of tick-transmitted lyme disease spirochete infection in a murine model. Antimicrobial agents and chemotherapy, 58(1), 348-351. https://doi.org/10.1128/AAC.01932-13

3. Tan HH, Mong R. Scorpion stings presenting to an emergency department in Singapore with special reference to Isometrus maculatus. Wilderness Environ Med. 2013 Mar;24(1):42-7. doi: 10.1016/j.wem.2012.10.001. Epub 2013 Jan 9. PMID: 23312559 . 